\title{
Evaluation of fibromyalgia syndrome in patients with rosacea
}

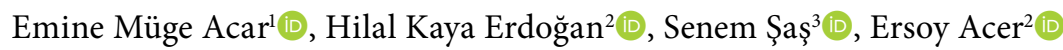 \\ ${ }^{1}$ Department of Dermatology and Venerology, Kırşehir Training and Research Hospital, Kırşehir, Turkey \\ ${ }^{2}$ Department of Dermatology and Venerology, Eskişehir Osmangazi University Faculty of Medicine, Eskişehir, Turkey \\ ${ }^{3}$ Department of Physical Medicine and Rehabilitation, Kırşehir Training and Research Hospital, Kırşehir, Turkey \\ ${ }^{4}$ Department of Dermatology and Venerology, Eskişehir Osmangazi University Faculty of Medicine, Eskişehir, Turkey
}

\begin{abstract}
Objectives: This study aims to investigate the frequency of fibromyalgia syndrome (FMS) in rosacea patients and the relationship between disease disability score of FMS and quality of life score of rosacea.

Patients and methods: This cross-sectional controlled clinical trial was performed between December 2017 and December 2018 . One hundred female rosacea patients (mean age 43.2 \pm 10.1 ; range, 21 to 65 years) and 100 age- and sex-matched control subjects (mean age $41.2 \pm 11.1 ;$ range, 22 to 68 years) with no history of skin disease and systemic diseases including diabetes, cardiovascular, renal and hepatic diseases were recruited. Dermatology Life Quality Index (DLQI) scores were calculated using a 10-item self-administered questionnaire. The diagnosis of FMS was established according to 2010 American College of Rheumatology diagnostic criteria. Fibromyalgia Impact Questionnaire (FIQ) was used to determine the clinical severity and functional disability, while Visual Analog Scale (VAS) was used to determine pain severity in the patients with FMS. Results: The frequency of FMS in patient group was significantly higher than control group ( $p=0.019)$. The mean duration of FMS in patient group was significantly higher than control group $(p=0.001)$. There was no significant difference in terms of the age of onset of FMS, FIQ and VAS scores between groups ( $p=0.53, p=0.54, p=0.07$, respectively). DLQI scores were significantly correlated with FIQ scores in the patient group $(r=0.43, p=0.008)$.

Conclusion: The frequency of FMS in rosacea patients was significantly higher than control subjects without any skin disease and there was a correlation between disability score of FMS and quality of life score of rosacea. Investigating fibromyalgia symptoms in rosacea patients may be helpful for providing patient-based therapeutic approaches where neurologically based treatments may also be beneficial for rosacea.

Keywords: Female, fibromyalgia, frequency, quality of life, rosacea.
\end{abstract}

Rosacea is a chronic inflammatory skin disorder with unknown etiology. Vascular abnormalities, environmental factors and microorganisms such as Demodex folliculorum and Helicobacter pylori have been documented to play a potential role in the development of the disease. ${ }^{1}$ Dysfunction of innate immune system, disruption of the epidermal skin barrier, activation of cutaneous nerves by triggering factors like sun exposure and emotional stress result with skin sensitivity. ${ }^{2-4}$ Coexistence of neurological and neuropsychiatric diseases with rosacea has been reported, supporting the role of neurophysiological mechanisms in disease etiopathogenesis. ${ }^{5,6}$

Fibromyalgia syndrome (FMS) is a chronic noninflammatory rheumatic disorder that is characterized by generalized musculoskeletal pain. ${ }^{7}$ Although the etiopathogenesis is not clear, the abnormalities in central pain mechanisms have been thought to play a crucial role. ${ }^{8}$

Received: June 14, 2020 Accepted: September 16, 2020 Published online: January 14, 2021

Correspondence: Emine Müge Acar, MD. Kırşehir Eğitim ve Araştırma Hastanesi, Dermatoloji ve Veneroloji Kliniği, 40200 Özbağ, Kırşehir, Türkiye. Tel: +90386 - 2134515 e-mail: drmugeacar@gmail.com 
Recently, the role of peripheral nerves and neurogenic inflammation has been implicated in the pathophysiology of FMS. ${ }^{9,10}$ Skin related symptoms including hyperhidrosis, burning sensation of the skin or mucous membranes and increased frequency of cutaneous disorders like neurotic excoriations, lichen simplex chronicus, dermographism, seborrheic dermatitis and tinea pedis have been reported in FMS patients. ${ }^{11}$ Rosacea is another skin disease which has been reported to accompany FMS. ${ }^{11}$ However, the relationship between rosacea and FMS has not been investigated as far as we are aware. Therefore, in this study, we aimed to investigate the frequency of FMS in rosacea patients and the relationship between disease disability score of FMS and quality of life score of rosacea.

\section{PATIENTS AND METHODS}

This cross-sectional controlled clinical trial was performed in Kırşehir Training and Research Hospital between December 2017 and December 2018. We included 130 consecutive female patients with rosacea. Thirty patients met the exclusion criteria and therefore were excluded from the study and a total of 100 patients were recruited. The control group was selected from our hospital staff by snowball technique and included 100 consecutive female controls. The mean ages of rosacea patients and control subjects were 43.21 (range, 21 to 65) years and 41.2 (range, 22 to 68) years, respectively $(p=0.18)$. Patients aged under 18 years, pregnant or lactating patients, those having a history of systemic or inflammatory diseases were excluded. The control group was selected from female subjects with no history of skin disease and systemic diseases including diabetes, cardiovascular, renal and hepatic diseases. The study protocol was approved by the Ahi Evran University Ethics Committee (approval no: 2017-18/219, date: 28.11.2017). A written informed consent was obtained from each participant. The study was conducted in accordance with the principles of the Declaration of Helsinki.

The patients were evaluated by a physical medicine and rehabilitation specialist and the diagnosis of FMS was established according to 2010 American College of Rheumatology diagnostic criteria. ${ }^{12,13}$ A validated Turkish version of the Fibromyalgia Impact Questionnaire (FIQ) was used to determine the clinical severity and functional disability of the patients diagnosed with FMS. The forms were filled out by the patients in the outpatient clinic and took approximately 5 min to complete. FIQ forms consist of 10 self-administered scales related to physical functioning, work status, depression, anxiety, sleep, pain, stiffness, fatigue and well being (scores between 0 to 80). Visual Analog Scale (VAS) (0 to 10) was used to determine the pain severity in FMS. ${ }^{14,15}$

Dermatology Life Quality Index (DLQI) scores were calculated using a 10-item self-administered questionnaire. The patients filled out the Turkish version of the DLQI form in approximately 3 to $4 \mathrm{~min}$ in the outpatient clinic. The DLQI consists of 10 questions regarding symptoms

Table 1. Characteristics of patient and control groups

\begin{tabular}{|c|c|c|c|c|c|c|c|}
\hline & \multicolumn{3}{|c|}{ Patient group $(n=100)$} & \multicolumn{3}{|c|}{ Control group $(n=100)$} & \multirow[b]{2}{*}{$p$} \\
\hline & $\mathrm{n}$ & $\%$ & Mean \pm SD & $\mathrm{n}$ & $\%$ & Mean \pm SD & \\
\hline Age (year) & & & $43.21 \pm 10.1$ & & & $41.2 \pm 11.1$ & 0.18 \\
\hline Rosacea duration (month) & & & $54.8 \pm 5.9$ & & & - & - \\
\hline Fibromyalgia age onset & & & $34.9 \pm 1.2$ & & & $36.4 \pm 2.4$ & 0.53 \\
\hline \multicolumn{8}{|l|}{ Subjective symptoms } \\
\hline Itching & 55 & 55 & & - & - & & - \\
\hline Burning sensation & 53 & 53 & & - & - & & - \\
\hline Stinging sensation & 11 & 11 & & - & - & & - \\
\hline
\end{tabular}




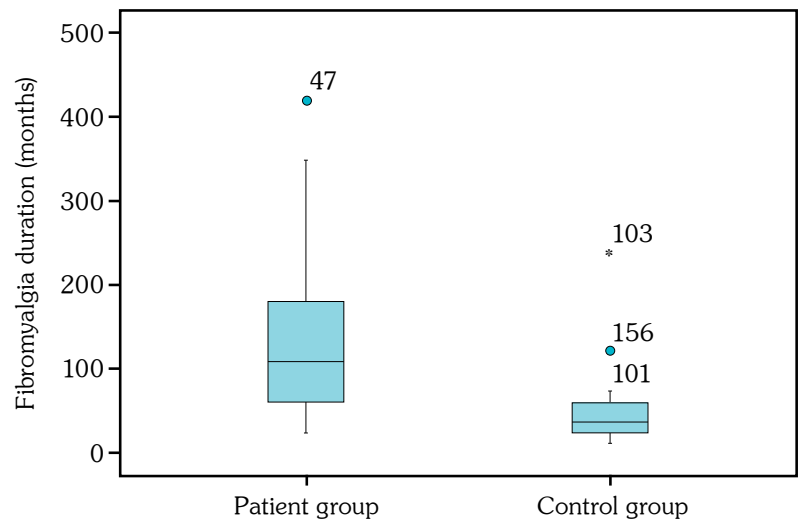

Figure 1. Comparison of fibromyalgia durations between patient and control groups.

and feelings, daily activities, leisure, work or school, personal relationships and treatment in the previous seven days. The questions have four alternative answers: not at all, a little, a lot and very much, which correspond to scores of $0,1,2$ and 3 , respectively. The total score is calculated by summing the score of each question ranging from a minimum of 0 to a maximum of $30 . .^{16,17}$

The age of patients, disease duration, skin types, symptoms like itching, burning and stinging sensations were recorded. Skin types were assessed based on Fitzpatrick scale. ${ }^{18}$ FMS symptoms like fatigue, sleep disturbances, cognitive difficulties and stiffness were recorded.

\section{Statistical analysis}

Statistical analysis was performed using the IBM SPSS version 21.0 software (IBM Corp., Armonk, NY, USA) and G*Power Version 3.1.9.6 (Kiel Universität, Kiel, Germany). Shapiro-Wilk test was used for testing normality. Continuous and discrete variables were presented as mean \pm standard deviation and median (min-max), respectively. Categorical variables were shown as number and percentage. Fisher's exact test was used for analyzing categorical variables. The statistical difference between the groups was analyzed using Student's t-test for mean values and Mann-Whitney $U$ test for median values. Correlation analysis was performed using Spearman's correlation test. $\mathrm{P}<0.05$ was considered to be statistically significant.

\section{RESULTS}

The mean age of onset of rosacea was $38.8 \pm 1.1$ years. Fifteen patients had a family history of rosacea. As for skin types; 24 were of skin type I, 47 were of skin type II and 29 were of skin type III. Fifty-five patients described itching, 53 burning and 11 stinging sensation (Table 1).

Thirty-seven (37\%) patients were diagnosed with FMS in patient group; 10 patients already had a diagnosis of FMS at the time of examination. Twenty-one (21\%) subjects were diagnosed with FMS in the control group. The frequency of FMS was significantly higher in the patient group than in the control group $(p=0.019)$. The mean duration of FMS was $138.5 \pm 17.0$ months in the patient group and $52.0 \pm 11.5$ in the control group $(p=0.001)$ (Figure 1). In $24(64.8 \%)$ of the patients FMS started before rosacea symptoms. The mean FIQ scores did not differ between groups. $(p=0.54)$ (Table 2). DLQI scores were significantly correlated with FIQ scores in the patient group. $(\mathrm{r}=0.39 ; \mathrm{p}=0.017)$ (Figure 2$)$.

Table 2. Dermatology Life Quality Index scores of patient group, Fibromyalgia Impact Questionnaire and Visual Analog Scale scores of patient and control groups

\begin{tabular}{|c|c|c|c|c|c|c|c|c|c|c|c|}
\hline & \multicolumn{5}{|c|}{ Patient group $(\mathrm{n}=100)$} & \multicolumn{5}{|c|}{ Control group $(n=100)$} & \multirow[b]{2}{*}{$p$} \\
\hline & $\mathrm{n}$ & $\%$ & Mean \pm SD & Median & Min-Max & $\mathrm{n}$ & $\%$ & Mean \pm SD & Median & Min-Max & \\
\hline DLQI score & & & & 9 & $1-30$ & & & & & - & - \\
\hline FIQ score & & & $54.9 \pm 3.0$ & & & & & $50.8 \pm 5.0$ & & & 0.54 \\
\hline Pain severity (VAS scale) & & & & 7 & $2-10$ & & & & 6 & $2-10$ & 0.07 \\
\hline
\end{tabular}




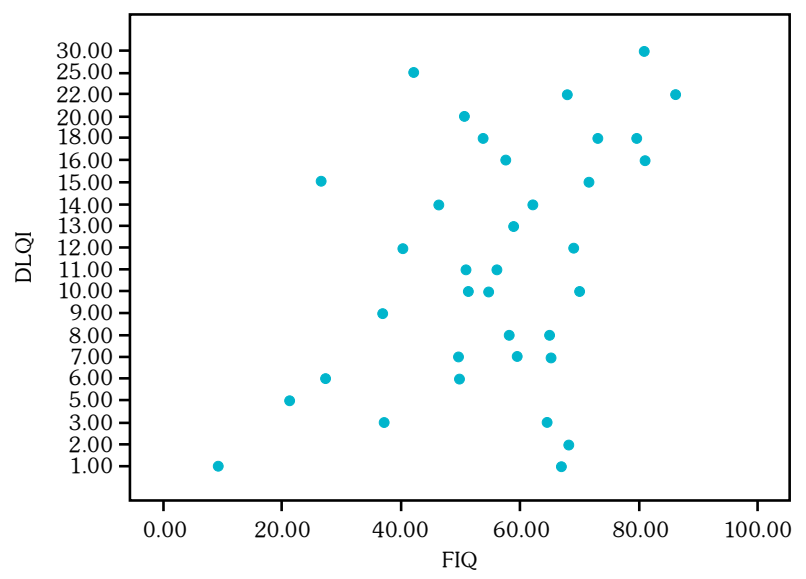

Figure 2. Correlation between Dermatology Life Quality Index and Fibromyalgia Impact Questionnaire in patient group.

DLQI: Dermatology Life Quality Index; FIQ: Fibromyalgia Impact Questionnaire.

\section{DISCUSSION}

The pathophysiology of rosacea has not yet been fully elucidated and is considered as multifactorial. ${ }^{19}$ Neuroimmune and neurovascular interactions are noted among the mechanisms in disease etiopathogenesis leading to flushing, erythema and induction of leukocyte infiltration. Rosacea has been reported to be accompanied by neurological and neuropsychiatric disorders including headaches, complex regional pain syndrome, essential tremor, depression and obsessive-compulsive disorder. ${ }^{5}$ Another striking coexistence with rosacea is migraine which is also a chronic paroxysmal disorder involving the trigeminal innervated area. ${ }^{2,6}$

Rheumatologic disorders including FMS, lupus, rheumatoid arthritis, mixed connective tissue disease and psoriatic arthritis are also reported to be common in rosacea patients. ${ }^{5}$ Dermatological disorders including dermographism, lichen simplex chronicus, tinea pedis and seborrheic dermatitis have been reported to be more common in FMS patients than the healthy population as well. ${ }^{20}$ FMS is a chronic pain syndrome characterized by generalized pain, fatigue, sleep disorders and cognitive function disorders. In our study, in which we evaluated the relationship between rosacea and FMS, we found that the frequency of FMS was higher in rosacea patients than controls and the frequency of FMS was higher in both patient and control groups than that of the normal population which is estimated to be around 0.5 to $5 \% .{ }^{21}$ This result may be related to the selection of the control group from our hospital staff who work under stressful conditions as work-related stress has been shown to contribute to the development of FMS. ${ }^{22,23}$ Recent studies have demonstrated the role of peripheral nerves and neurogenic inflammation in FMS. ${ }^{9}, 10$ Increased frequency of FMS in rosacea patients may be attributed to the common role of neurogenic inflammation in which substance $P$, mast cells, interleukin-1 beta and tumor necrosis factor-alpha are involved in both diseases. ${ }^{4,24-28}$

On the other hand, the similarity of the triggering factors of rosacea and FMS is noteworthy. FMS patients are highly sensitive to various environmental factors including heat, light, cold, chemical exposure, sound and smell, most of which also affect rosacea symptoms. ${ }^{24}$ Furthermore, both rosacea and FMS can be aggravated with consumption of certain foods and food additives such as caffeine, chocolate and glutamate. ${ }^{29-33}$

Fibromyalgia syndrome is also closely associated with stress, which is another triggering factor for rosacea. ${ }^{22,23}$ In addition, some skin changes including reticular skin discoloration, pressure hyperalgesia, reactive skin hyperemia and Raynauds phenomenon are seen in FMS supporting that neurovascular changes are not only limited to musculoskeletal system but they also affect the skin. ${ }^{34}$

In our study, the FMS duration in the patient group was significantly higher than that of the control group. This finding gives an impression that long-term exposure to neuromediators may cause a tendency to develop rosacea. The FIQ and VAS values did not differ between groups suggesting that the presence of rosacea was not associated with the increase in the severity of FMS. However, FIQ scores correlated with DLQI scores; this suggests that common pathophysiological mechanisms such as neuroinflammation may be involved in FMS and rosacea.

In addition to the four rosacea subtypes (erythematotelangiectatic, papulopustular, phymatous and ocular types), Scharschmidt et al., ${ }^{5}$ described the term neurogenic rosacea as 
another subgroup which is refractory to classical treatments and necessitates neurologically based treatment approaches including gabapentin, duloxetine, pregabalin, tricyclic antidepressants or topical doxepin, glycopyrrolate, capsaicin and ketamine. Gabapentin, duloxetin and tricyclic antidepressants are also used in FMS treatment, which supports that there are common therapeutic targets in the treatment of both diseases.

In this study, whether patients with FMS were using such medications at the time of the study, which can also affect rosacea symptoms, was not evaluated in detail and this is a limitation. The high frequency of FMS in rosacea patients in our study can be explained by the role of common pathophysiological mechanisms in both diseases.

In conclusion, in this study, we found that the frequency of FMS in rosacea patients was significantly higher than control group without skin disease and there was a correlation between DLQI and FIQ scores, suggesting a close relationship between FMS and rosacea. Questioning rosacea patients for FMS symptoms may be helpful for planning a patient-based treatment approach where neurologically based treatments may also be beneficial for rosacea. Further studies on the pathophysiological relationship of rosacea and FMS are warranted.

\section{Declaration of conflicting interests}

The authors declared no conflicts of interest with respect to the authorship and/or publication of this article.

\section{Funding}

The authors received no financial support for the research and/or authorship of this article.

\section{REFERENCES}

1. Crawford GH, Pelle MT, James WD. Rosacea: I. Etiology, pathogenesis, and subtype classification. J Am Acad Dermatol 2004;51:327-41.

2. Aubdool AA, Brain SD. Neurovascular aspects of skin neurogenic inflammation. $J$ Investig Dermatol Symp Proc 2011;15:33-9.

3. Dirschka T, Tronnier H, Fölster-Holst R. Epithelial barrier function and atopic diathesis in rosacea and perioral dermatitis. Br J Dermatol 2004;150:113641.
4. Gerber PA, Buhren BA, Steinhoff M, Homey B. Rosacea: The cytokine and chemokine network. J Investig Dermatol Symp Proc 2011;15:40-7.

5. Scharschmidt TC, Yost JM, Truong SV, Steinhoff M, Wang KC, Berger TG. Neurogenic rosacea: a distinct clinical subtype requiring a modified approach to treatment. Arch Dermatol 2011;147:123-6.

6. Christensen CE, Andersen FS, Wienholtz N, Egeberg A, Thyssen JP, Ashina M. The relationship between migraine and rosacea: Systematic review and metaanalysis. Cephalalgia 2018;38:1387-98.

7. Weigent DA, Bradley LA, Blalock JE, Alarcón GS. Current concepts in the pathophysiology of abnormal pain perception in fibromyalgia. Am $\mathrm{J}$ Med Sci 1998;315:405-12.

8. Abeles AM, Pillinger MH, Solitar BM, Abeles M. Narrative review: the pathophysiology of fibromyalgia. Ann Intern Med 2007;146:726-34.

9. Kim SH, Kim DH, Oh DH, Clauw DJ. Characteristic electron microscopic findings in the skin of patients with fibromyalgia--preliminary study. Clin Rheumatol 2008;27:407-11.

10. Eneström S, Bengtsson A, Frödin T. Dermal IgG deposits and increase of mast cells in patients with fibromyalgia--relevant findings or epiphenomena? Scand J Rheumatol 1997;26:308-13.

11. Laniosz V, Wetter DA, Godar DA. Dermatologic manifestations of fibromyalgia. Clin Rheumatol 2014;33:1009-13.

12. Wolfe F, Clauw DJ, Fitzcharles MA, Goldenberg DL, Häuser W, Katz RL, et al. 2016 Revisions to the 2010/2011 fibromyalgia diagnostic criteria. Semin Arthritis Rheum 2016;46:319-29.

13. Bennett R. The Fibromyalgia Impact Questionnaire (FIQ): a review of its development, current version, operating characteristics and uses. Clin Exp Rheumatol 2005;23(5 Suppl 39):S154-62.

14. Ediz L, Hiz O, Toprak M, Tekeoglu I, Ercan S. The validity and reliability of the Turkish version of the Revised Fibromyalgia Impact Questionnaire. Clin Rheumatol 2011;30:339-46.

15. Crichton N. Visual analogue scale (VAS). J Clin Nurs 2001;10:706-6.

16. Finlay AY, Khan GK. Dermatology Life Quality Index (DLQI)--a simple practical measure for routine clinical use. Clin Exp Dermatol 1994;19:210-6.

17. Oztürkcan S, Ermertcan AT, Eser E, Sahin MT. Cross validation of the Turkish version of dermatology life quality index. Int J Dermatol 2006;45:1300-7.

18. Sachdeva S. Fitzpatrick skin typing: applications in dermatology. Indian $\mathrm{J}$ Dermatol Venereol Leprol 2009;75:93-6.

19. Picardo M, Eichenfield LF, Tan J. Acne and Rosacea. Dermatol Ther (Heidelb) 2017;7(Suppl 1):43-52.

20. Erdogan HK, Sas S, Acer E, Bulur I, Altunay IK, Erdem HR. Cutaneousfindings infibromyalgia syndrome and their effect onquality of life. Dermatologica Sinica 2016;34:131-4. 
21. White KP, Harth M. Classification, epidemiology, and natural history of fibromyalgia. Curr Pain Headache Rep 2001;5:320-9.

22. Kivimäki M, Leino-Arjas $P$, Virtanen $M$, Elovainio M, Keltikangas-Järvinen L, Puttonen S, et al. Work stress and incidence of newly diagnosed fibromyalgia: prospective cohort study. J Psychosom Res 2004;57:417-22.

23. $\mathrm{Su} \mathrm{D}$, Drummond PD. Psychological stress and rosacea. In: Whitehead J, Barrows WB, editors. Journal of the Rosacea Research \& Development Institute. New York: iUniverse Inc; 2010. p. 32.

24. Staud R. Biology and therapy of fibromyalgia: pain in fibromyalgia syndrome. Arthritis Res Ther 2006;8:208.

25. Kulka M, Sheen CH, Tancowny BP, Grammer LC, Schleimer RP. Neuropeptides activate human mast cell degranulation and chemokine production. Immunology 2008;123:398-410.

26. Bengtsson A. The muscle in fibromyalgia. Rheumatology (Oxford) 2002;41:721-4.

27. Muto Y, Wang Z, Vanderberghe M, Two A, Gallo RL, Di Nardo A. Mast cells are key mediators of cathelicidin-initiated skin inflammation in rosacea. $\mathrm{J}$ Invest Dermatol 2014;134:2728-36.
28. Blanco I, Béritze N, Argüelles M, Cárcaba V, Fernández $\mathrm{F}$, Janciauskiene $\mathrm{S}$, et al. Abnormal overexpression of mastocytes in skin biopsies of fibromyalgia patients. Clin Rheumatol 2010;29:1403-12.

29. Holton KF, Taren DL, Thomson CA, Bennett $\mathrm{RM}$, Jones KD. The effect of dietary glutamate on fibromyalgia and irritable bowel symptoms. Clin Exp Rheumatol 2012;30(6 Suppl 74):10-7.

30. Harte S, Hassett A, Brummett C, Harris R, Clauw D. Daily caffeine consumption is associated with fibromyalgia pain. The Journal of Pain 2012;13:S32.

31. JaworekAK, Wojas-Pelc A, Pastuszczak M. Aggravating factors of rosacea. Przegl Lek 2008;65:180-3.

32. Kaartinen K, Lammi K, Hypen M, Nenonen M, Hanninen O, Rauma AL. Vegan diet alleviates fibromyalgia symptoms. Scand $J$ Rheumatol 2000;29:308-13.

33. Tüzün Y, Wolf R, Kutlubay Z, Karakuş O, Engin B. Rosacea and rhinophyma. Clin Dermatol 2014;32:35-46.

34. Granges G, Littlejohn GO. A comparative study of clinical signs in fibromyalgia/fibrositis syndrome, healthy and exercising subjects. J Rheumatol 1993;20:344-51. 